\title{
RTAEB: Reliable Traffic Aware Energy Balance Routing in Wireless Sensor Networks
}

\author{
Fatma H. El-Fouly, Rabie A. Ramadan, Mohamed I. Mahmoud, and Moawad I. Dessouky
}

\begin{abstract}
This paper proposes a Reliable Traffic Aware Energy Balance greedy Algorithm (RTAEB) to achieve both minimum energy consumption and balanced energy consumption among sensor nodes for Wireless Sensor Network (WSN) lifetime extension. In addition, data reliability is considered in our model where, the sensed data can reach the sink node in a more reliable way. Finally, buffer space is considered to reduce the packet loss and energy consumption due to the retransmission of the same packets. Through simulation, the performance of proposed algorithm is compared with the previous work such as EBRP, ACO, TADR, SEB, and CLR-Routing.
\end{abstract}

Keywords -WSNs, routing, energy balancing, reliability.

\section{Introduction}

Communication in WSNs, communication has been recognized as the major source of energy consumption and costs significantly more than computation [1]. Consequently, most of the existing routing techniques in WSN attempt to find the shortest path to the sink to minimize energy consumption. As a result, highly unbalanced energy consumption which causes energy holes around the sink and significant network lifetime reduction [2]. Therefore, designing energy-balanced routing technique plays a crucial role in WSNs [2].

The reliable data transmission is one of the most essential issues in WSNs [3]. The loss of important information due to unexpected node failure or dynamic nature of wireless communication link [4] prevents the sensor network from achieving its primary purpose which is data transfer. Hence,

Fatma H. Elfouly

Department of Electronics and Electrical Communications, Higher Institute of Engineering, El-Shorouk Academy

Cairo, Egypt

Rabie A. Ramadan

Department of Computer Engineering, Faculty of Engineering, Cairo

University, On leave at Hail University y, Hail, KSA.

Cairo, Egypt

Mohamed I. Mahmoud

Department of Control Engineering and Industrial Electronics, Faculty of Electronic Engineering, Menoufia University.

Egypt

\section{Moawad I. Dessouky}

Department of Electronics and Electrical Communications Egypt routing techniques should give priority to reliable transmission .At the same time, it is critical to reduce packet loss in WSNs which will improve the network throughput and energyefficiency.

Due to memory constraints on sensor nodes, buffering a large number of packets is impossible. Thus, such a buffer overflow problem may result in information loss and more energy consumption due to the retransmission of the same packets. Thus, such retransmission limits the network's

lifetime and efficiency. Consequently, it is a highly needed to consider buffer space when designing routing protocols in WSNs [5].

The problems of energy balance and reliable communication have received significant attention in recent years $[2,3]$. However, our contributions in this paper focus on: 1) reducing and balancing energy consumption among sensor nodes for WSN lifetime extension, well, 2) enhancing data reliability where the sensed data can reach the sink node in a more reliable way, 3) Taking into consideration buffer space on sensor nodes, and 4) Introducing a RTAEB heuristic algorithm based energy reduction and reliability as well as load balancing.

The rest of this paper is organized as follows: section II introduces a brief summary of the related work. Section III introduces the problem description. Then, section IV describes the RTAEB based approach. Section V provides the simulation results. Finally, Section VI concludes the paper.

\section{Related Work}

This section focuses only on the most related work to the proposal of this paper. It starts by explaining the work presented in $[2,6,5,7,8]$ followed by the differences from our proposal.

[2] Proposed an Energy-Balanced Routing Protocol (EBRP). EBRP algorithm borrows the concept of potential in physics to construct a mixed virtual potential field in terms of depth, energy density, and residual energy. [6] Proposed an improved ant colony optimization routing (ACO) for WSN to prolongs network lifetime. The ACO improved approach in [6] enhanced an approach based on ACO by adding new heuristic information to distinguish the best neighbor.

Meanwhile, the analysis of ACO improved algorithm [6], and EBRP [2] show that some issues are not considered which are reflected as drawbacks. Firstly, the network reliability, as 
discussed above, this might affect the network efficiency. The second is the queue buffer size in which it has directly impact on network throughput and lifetime. Finally, node load where, taking residual energy only into consideration as in $[2,6]$ is not sufficient to achieve balanced energy usage in the network.

[5] Proposed a traffic aware dynamic routing (TADR) algorithm to avoid congestion. In this algorithm, a hybrid potential field is constructed in terms of depth and the normalized queue length. However, TADR algorithm doesn't consider two critical issues which regard as a drawback. The first is energy balancing, as described above; this might cause energy holes around the sink and significant network lifetime reduction. The second issue is the network reliability. This might deteriorate the network performance as mentioned above.

[7] Proposed a simple Cross-Layer Balancing Routing (CLB-Routing) that enhances the WSNs lifetime by balancing the energy consumption in the forwarding task. The choice of the next hop is according to a probability, which counts residual energy, energy of communication, and the number of times that each forwarding node has routed data. However, CLB-Routing had important issues to take into account, but it lacked some others like network reliability and buffer size. This eventually affect the network throughput and lifetime as described above.

[8] Proposed a Swarm intelligence based energy balance routing scheme (SEB). It balances residual energy on sensor nodes evenly according to their weights as much as possible. The probability of selecting the next hop neighbor node is calculated according to residual energy, distance to the sink, weight of nodes, and the environment pheromone which is related to path quality. Nevertheless, the previous study of SEB shows that it has a drawback since some issues are not considered. The first is the packet buffer capacity of sensor nodes. As described above, this inevitably affects the network efficiency. Secondly, the dynamic behavior of the wireless link quality over time and space This can be easily lead to the use of low-quality links, and result in unreliable routs [9].

The proposed RTAEB algorithm in this paper considers the end-to-end reliability of a multi-hop route based on the Packet Reception Rate (PRR) which is one of the most commonly used reliability metrics [10]. Moreover, the proposed algorithm can balance energy consumption among sensor nodes evenly as much as possible through new effective function between nodes residual energy and weight. As well as, it can effectively alleviate buffer overflow by integrating the normalized buffer space into routing choice.

\section{Problem Description}

Consider a static multi-hop WSN deployed in the sensing field. In this model, we aim to achieve reliable routing algorithm taking into consideration nodes energy consumption, energy balancing among sensor nodes, and nodes buffer space. The WSN can be modeled as a random geometric graph, $G(V, L)$, where $V$ denotes the set of sensor nodes which distributed randomly in the square monitoring field and $L$ represents a set of all communication links $(i, j)$ where, $i, j \in V$. Link $(i, j)$ exists if and only if nodes $i, j$ are within radio range of each other. The events in the environment will be detected by some sensor nodes which are called source nodes. Assuming that the MAC layer provides the link quality estimation service, e.g., the $P R R$ information on each link [11], where each node is aware of the $P R R$ values to its one-hop neighbors. The information regarding the presence of the detected events at each source node should be reported to the sink node through intermediate sensor nodes which acts as a relay nodes. The chosen path from each source node to the sink should be the best path which satisfies some constraints including 1) low communication cost, 2) its reliability greater than or equal target value, 3) at the same time, sensor nodes on that path should have the maximum value resulting from a new proposed equation between the residual energy and weight compared with their neighbors to balance energy consumption among sensor nodes, and 4) as well, sensor nodes should have a buffer space greater than or equal message size to reduce packet loss and energy consumption due to retransmission of the same packets as a result of buffer overflow.

To simplify the description of the problem and its formulation, the notations used to model the problem are given in Table I.

TABLE I. OUR MODEL NOTATIONS

\begin{tabular}{|c|c|}
\hline \multicolumn{2}{|r|}{ Given Parameters } \\
\hline Notation & Description \\
\hline$R E_{j}$ & The residual energy of each sensor node $j$ \\
\hline$s e_{(i, j)}$ & $\begin{array}{l}\text { The energy required to do single hop transmission from } i \text { to } j \text {, } \\
(i, j) \in L \text {. }\end{array}$ \\
\hline $\operatorname{Mes}_{i}$ & The number of messages at node $i$, \\
\hline$h_{c j}$ & The hop count of node $j$ \\
\hline$E w r_{j}$ & $\begin{array}{l}\text { The residual energy to weight ratio for each neighbor node } j \text {, } \\
j \in N E B_{i}, N E B_{i} \in S \cup R\end{array}$ \\
\hline$E N C_{j}(t)$ & $\begin{array}{l}\text { The ratio between residual energy to initial energy for each } \\
\text { neighbor node } j \text { at time } t\end{array}$ \\
\hline$p z$ & The packet size. \\
\hline$b s_{j}(t)$ & Buffer space in node $j$ at time $t$ \\
\hline$b m_{j}(t)$ & The normalized buffer space of node $j$ at time $t$ \\
\hline$N R E_{j}$ & The ratio between $R E_{j}$ and $s e_{(i, j)}$ for each neighbor node $j$, \\
\hline$N E B_{i}$ & The set of neighbors of node $i$ \\
\hline
\end{tabular}

\section{Iv. RTAEB Based Approach}

This section describes the details of the proposed RTAEB greedy algorithm for energy balance and reliable routing in WSNs. The proposed RTAEB algorithm is composed of two phases. In the first phase, nodes collect the information of their neighbor nodes and the minimum hop count from each one of them to the sink using broadcasting technique. In the second phase, the source nodes detect the events in the environment and send their data to the sink through the relay nodes. The 
selection of the relay node is related to the node cost. The node having the maximum cost is to be considered as the next hop node.

\section{A. Problem Formulation}

In order to achieve energy balanced routing, the node with heavy weight and low residual energy should be prevented from being selected as a next hop. So, the proposed algorithm considers a model in which the sensor node residual energy and weight are used when choosing the relay node through a new proposed function.

Now, let's start with the computation of the weight of a neighbor $j$ at time $t$ by equation (1).

$$
w e_{j}(t)=\left\{\begin{array}{cl}
\sum_{i \in N E B_{j}} \operatorname{Mes}_{i}(\mathrm{t}) & \text { if } h \mathrm{c}_{j} \leq h c_{i} \\
0 & \text { otherwise }
\end{array}\right.
$$

Node weight can be defined as the total number of messages at its neighbor nodes which may choose it to relay their messages. Equation (1) means that packets are not allowed to be transmitted backward to the neighbors with higher hop count. This strategy ensures that the packets are forwarded closer toward the sink and prevents forming a loop.

In addition, the new function that combines residual energy and weight for each node $j$ at time $t$ is defined by equation (2) as follows:

$$
E w r_{j}(t)=\left\{\begin{array}{c}
\left(\left(N R E_{j}(t)-w e_{j}(t)\right)+1\right) \cdot\left(\exp \left(E N C_{j}(t)\right)\right) \text { if } N R E_{j}(t) \geq w e_{j}(t) \\
\left(\frac{1}{w e_{j}(t)-N R E_{j}(t)}\right) \cdot\left(\exp \left(E N C_{j}(t)\right)\right) \quad \text { if } N R E_{j}(t)<w e_{j}(t) \\
0 \quad \text { if } w e_{j}(t)=0
\end{array}\right.
$$

During the multi-hop transmission the relay node needs to hold in a buffer the incoming data packets during the processing time required for the previous ones. The sensor nodes have limited memory, it is impossible to buffer a large number of packets. Consequently, the buffer of the relay node may start overflowing, resulting in loss of important packets and more energy consumption due to the retransmission of the same packets [12]. For efficient use of available buffer, we consider a model in which the probability of buffer overflow is minimized as much as possible by integrating the normalized buffer space into routing choice. The normalized buffer space is defined as the ratio between the buffer space and packet size. It is used to express the number of packets that can be received by every sensor node without it starting buffer overflowing at a certain time. The normalized buffer space of node $\mathrm{j}$ at time $\mathrm{t}$ can be defined as follows:

$$
b m_{j}(t)=\left\{\begin{array}{cl}
\frac{b s_{j}(t)}{p z} & \text { if } b s_{j}(t) \geq p z \\
0 & \text { otherwise }
\end{array}\right.
$$

Whenever, a given node $i$ have data to send, it calculates the value of $N C_{j}$ associated for each neighbor node $j$. Then, it sends its data over the neighbor node having the greater value of $N C_{j}$ which is calculated as follows:

$$
N C_{j}(t)=e_{j}(t)+r_{i j}(t)+h d_{i j}(\mathrm{t})+b m_{j}(t)
$$

Where

$$
\begin{gathered}
e_{j}(t)=\frac{E w r_{j}(t)}{E_{i n_{j} * \sqrt{h c_{j}}}} \\
r_{i j}(t)=P R R_{i j}+\frac{P R R_{i j} * P R R_{s i}}{\alpha+\left(Q-\left(P R R_{i j} * P R R_{s i}\right)\right)} \\
h d_{i j}(t)=\frac{\left(h c_{i}-h c_{j}\right)+1}{1+\sqrt{\left(h c_{j}+h c_{s i}+1\right) / h c_{j}}}
\end{gathered}
$$

Where, $P R R_{s i}, h c_{s i}$ represents the packet reception ratio and the number of hops from the source node $s$ to the node $i$ respectively, $E_{i n_{j}}$ is the initial residual energy of node $j$, and $\alpha$ is a constant.

In order to avoid or reduce packet loss due to buffer overflow which in turn improve the overall network performance, it is critical to send packets to the sensor node with more buffer space or less traffic load. Therefore, $b m j(t)$ is used in the calculation of $N C_{j}$ which enables decision making according to the buffer apace on the neighbor nodes.

As in equation (5), $e_{j}(t)$ is related to the proposed relation between residual energy and weight and The root mean square of the hop count. $E w r_{j}(t)$ is used in order to maintain higher and balance residual energy on sensor nodes. Selecting the nodes which have the largest residual energy compared to its weight may result in more delay because the node which is far away from the sink node may be chosen as the next hop. Therefore, the square root of the hop count is used in the calculation of $e_{j}(t)$. The reason why the square root is used in the calculation of $e_{j}(t)$ lies in the fact that the value of this function will become steeper by the decrease of hop count. The use of $E_{i n_{j}}$ for normalization.

Since energy conservation is an essential issue in WSN, selecting the nodes with minimum hop count is required to minimize energy consumption and conserve much more energy as possible. Therefore, the function $h d_{i j}(t)$ is used in the calculation of $N C_{j}$. Finally, to improve network reliability, data packet should be transmitted on a more reliable way to the sink. So, the reliability function $r_{i j}(t)$ is used in the calculation of $N C_{j}$.

\section{B. Description of RTAEB Algorithm}

During the initialization phase, the sink node broadcasts a control message to all 1-hop neighbors with a hop count field initialized to " 0 ". Each node receiving the message updates its hop count (increments the value by 1 and rebroadcasts the control message to its 1-hop neighboring nodes. If any node receives the control message from the node whose hop count is lower or equal its hop count. Then the sending node is considered as the next hop candidate node; otherwise, the control message is discarded. This process repeated until all the nodes are initialized. 
Once the source node detects an event, the process of selecting the relay nodes for data transmission is started. If any node i having data to send, it broadcasts the next hop selection message to its 1-hop neighbors in order to collect the information required to calculate the value of $e_{j}(t), r_{i j}(t)$, and $b m_{j}(t)$. On receiving the reply, if the buffer space of any neighbor node of $i$ is found to be less than the required packet size or the hop count of the neighbor node is found to be greater than the hop count of the node itself, the message is discarded. Then this neighbor node is removed from the routing calculation. Once the reply message is received, the node $i$ calculates the link cost using equation (4) and selects the neighbor node having the maximum cost. The pseudo code of RTAEB algorithm is given as Algorithm 1.

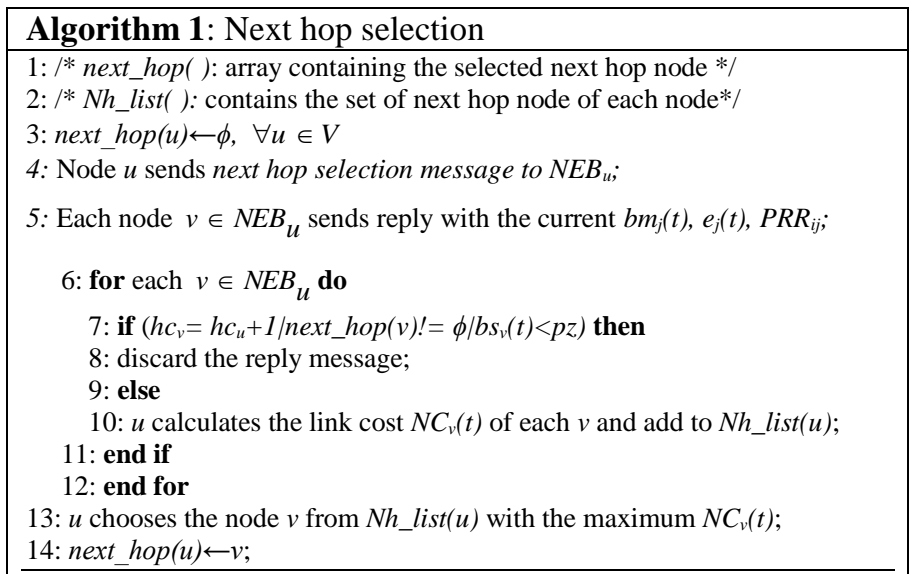

\section{v. Performance Evaluation}

In this section, different experiments are conducted to evaluate the performance and validate the effectiveness of our proposal. The section starts by describing the performance metrics followed by simulation environment and finally simulation results.

\section{A. Performance Metric}

For a comprehensive performance evaluation, several quantitative metrics considered are defined below.

1. Network Lifetime [2]. It is defined as the time duration from the begging of the network operation until the first node exhausts its battery.

2. Throughput Ratio (TR) [9]. This metric is defined as:

$$
T R=\frac{\text { Number of packetsreceivedby the } \sin k}{\text { Number of packetssent by sourcenodes }}
$$

3. Average End-to-End Delay (Seconds) [13]: It is defined as the average time a packet takes to travel from source node to the sink node. This includes propagation, transmission, queuing, and processing delay. The processing delay can be ignored as a result of fast processing speed .

\section{B. Simulation Environment}

In this paper, the simulation environment consists of 80 sensor nodes deployed randomly in a field. The sink node, and sensor nodes are stationary after being deployed in the field. Furthermore, the sink node is located at $(1000,500) \mathrm{m}$. All the later experiments are done on a custom Matlab simulator. Data traffic is generated according to a passion process with mean parameter $\sigma$. In addition, we choose a harsh wireless channel model, which includes shadowing and deep fading effects, as well as the noise [14]. The simulation parameters are listed in Table II.

TABLE II. SIMULATION ENVIRONMENT PARAMETERS

\begin{tabular}{|l|l|}
\hline \multicolumn{1}{|c|}{ Parameters } & \multicolumn{1}{c|}{ values } \\
\hline Network size & $1000 \times 1000$ \\
\hline Number of nodes & 80 \\
\hline Node placement & Random uniform \\
\hline Packet size & 64 byte \\
\hline Frequency & $2400 \mathrm{MHz}$ \\
\hline Transmission power & $-5 \mathrm{dBm}$ \\
\hline Initial energy & $125 \mathrm{~mJ}$ \\
\hline Maximum transmission range & $223 \mathrm{~m}$ \\
\hline Channel model & Log-normal shadow \\
\hline Path loss exponent & 6 \\
\hline Shadow fading variance & 6 \\
\hline Noise power & $-145 \mathrm{dBm}$ \\
\hline Reference distance & $3 \mathrm{~m}$ \\
\hline
\end{tabular}

\section{Simulation Results}

To verify the feasibility and effectiveness of our proposal, its performance is compared in terms of Network Lifetime, End-to-End Delay, and Throughput Ratio, with the proposed protocols in $[2,6,5,7,8]$

\section{a) Network Lifetime Evaluation}

In this set of experiments the performance of the proposed RTAEB approach is evaluated in terms of network lifetime compared to EBRP [2], ACO proposed in [6], TADR [5], CLR-Routing [7], and SEB [8] under different traffic rate $\sigma$. Figure 1 shows the variation of network lifetime with respect to different traffic rate $\sigma$. From the figure it can be found that as the value of $\sigma$ increases, the network lifetime decreases. Since the network traffic increases with the increment of $\sigma$, the relay load of nodes increases linearly. This increase of load among nodes is the main reason behind decrease of lifetime. However, the figure shows clearly that the proposed RTAEB algorithm enhances significantly the network lifetime compared with the others. This means that the proposed RTAEB algorithm balances the network energy consumption more effectively than the others.

\section{b) Network Reliability Evaluation}

In this experiment, the performance of the proposed RTAEB approach is evaluated in terms of TR for both homogenous and heterogeneous networks compared to EBRP [2], ACO proposed in [6], TADR [5], CLR-Routing [7], and SEB [8] under different traffic rate $\sigma$. The TR against different traffic rate $\sigma$ is depicted in Figure 2. Clearly, the proposed RTAEB algorithm achieves the highest TR compared to the 
others and very close to the target value. This is because it forwards the data packets toward the sink in a more reliable way and alleviates the possible buffer overflow.

\section{c) Average End-to-End Delay Evaluation}

In this set of experiments, the performance of the proposed RTAEB approach is evaluated in terms of end-to-end delay compared to EBRP [2], ACO proposed in [6], TADR [5], CLR-Routing [7], and SEB [8] under different traffic rate $\sigma$. Figure 4 shows the changes in end-to-end delay against different traffic rate $\sigma$. From the results, it is observed that the end-end-delay increases, as the traffic rate increases. A higher traffic rate causes more queuing delay, which raises the endto-end delay. However, it is clear that the proposed RTAEB approach giving the lowest end-to-end delay compared with the others. This is because, the proposed RTAEB approach forwards the data packets toward the sink in a more reliable way and alleviates the possible buffer overflow, which decreases the packet loss and retransmissions and hence the end-to-end delay.

\section{vI. Conclusions}

In this work we presented an efficient RTAEB routing algorithm for WSNs. The proposed not only reduces the energy consumption but also balanced it among sensor nodes to extend WSN lifetime. At the same time, the sensed data delivered to the sink with the highest possible reliability and minimum buffer overflow. The performance of proposed method compared with the previous works which are related to our topic such as EBRP, ACO, TADR, SEB, and CLRRouting are evaluated and analyzed through simulation. Simulation results showed that our approach is robust; achieve longer network lifetime, and giving lower end-to-end delay compared to the previous works.

\section{References}

[1] G.J. Pottie and W.J. Kaiser, " Wireless Integrated Network Sensors," Communications of ACM, Vol. 43, No. 5, pp. 51-58, 2000.

[2] F. Ren, J. Zhang, T. He, C. Lin, and S. K. Das, "EBRP: EnergyBalanced Routing Protocol for Data Gathering in Wireless Sensor Networks," IEEE Trans. on Parallel and Distributed Systems, Vol. 22, No. 12, December 2011.

[3] X. Liu, "A transmission scheme for wireless sensor networks using ant colony optimization with unconventional characteristics," IEEE Communications Letters, Vol. 18, No. 7, pp. 1214-1217, 2014.

[4] J. Niu, L. Cheng, Y. Gu, L. Shu, and S. Das, "R3E: reliable reactive routing enhancement for wireless sensor networks," IEEE Transactions on Industrial Informatics, Vol. 10, No. 1, pp. 784-794, 2014.

[5] F. Ren, S. K. Das, and C. Lin, "Traffic-Aware Dynamic Routing to Alleviate Congestion in Wireless Sensor Networks," IEEE Transactions on Parallel and Distributed Systems, Vol. 22, No. 9, September 2011.

[6] A. El Ghazi, B. Ahiod, and A. Ouaarab, "Improved Ant Colony Optimization Routing Protocol for Wireless Sensor Networks," in P. G. Noubir and M. Raynal (Eds.): NETYS 2014, pp. 246-256, Springer, Heidelberg, 2014.

[7] S. Yaessad, L. Bouallouche, and D. Aissani, "A Cross-Layer Routing Protocol for Balancing Energy Consumption in Wireless Sensor Networks" Wireless Pers. Commun., V ol. , pp., Springer, 2014.

[8] D. Qian, H. Chen, W. Wu, and L. Cheng, "Swarm Intelligence Based Energy Balance Routing For Wireless Sensor Networks", proc. of the 2nd Int. Symposium on Intelligent Information Technology Application, vol. 2, pp.811-815, 2008.

[9] X. Baoshu, and W. Hui, "A reliability transmission routing metric algorithm for wireless sensor network", proc. IEEE Int. Conf. E-Health Networking, Digital Ecosystems and Technologies (EDT), Vol.1, pp.454 $-457,2010$.

[10] S. B. Kootkar, "Reliable sensor networks", M.S. thesis, Dept. Comp. Eng., TU Delft Univ., Delft, Netherlands, 2008.

[11] L. Cheng, J. Nia, J. Cao, S. K. Das, and Y. Gu, "QoS Aware Geographic Opportunistic Routing in Wireless Sensor Networks", IEEE Trans. On Parallel and Distributed Systems, 2014.

[12] O. Fdili, Y. Fakhri, and D. Aboutajdine, "Impact of queue buffer size awareness on single and multi service real-time routing protocols for WSNs," International Journal of Communication Networks and Information Security, Vol. 4, pp. 104-111, 2012.

[13] V. K. Verma, S. Singh, and N. P. Pathak, "Analysis of scalability for AODV routing protocol in wireless sensor networks," OptikInternational Journal for Light and Electron Optics, vol. 125, no. 2, pp. 748-750, 2014.

[14] “MicaZ wireless module." [Online]. Available: http://www.xbow.com/ Products/ Product pdf files/Wireless pdf/MICAzDatasheet.pdf.

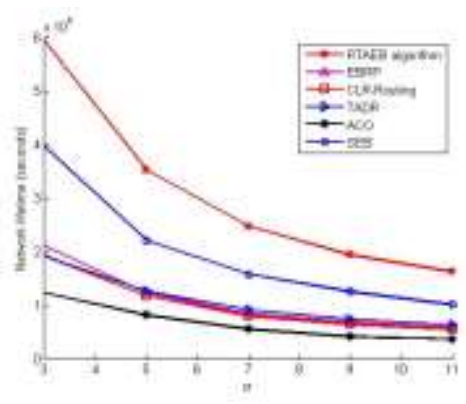

Figure 1. Network lifetime vs. traffic rate $\sigma$

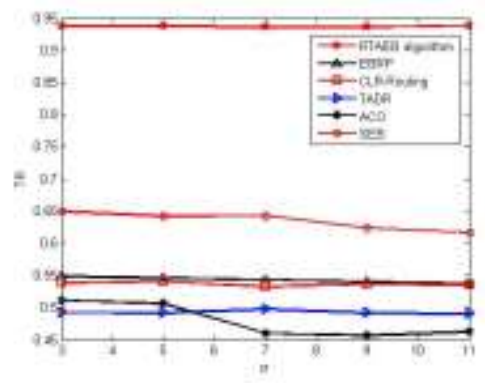

Figure 2. Network throughput vs. traffic rate $\sigma$

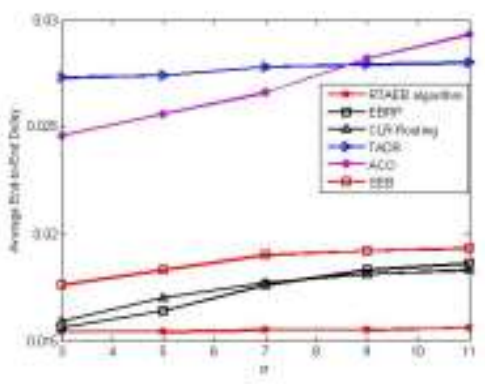

Figure 3. Average end-to-end delay vs. traffic rate $\sigma$ 